\title{
The Daily Resistive Index measurement useful tool in the estimation of the optimal time interval between two Shock Wave Lithotripsy sessions.
}

\author{
Esin Yencilek ${ }^{1}$ Aysegul Sarsilmaz ${ }^{2}$, Ozgur Kilickesmez ${ }^{3}$, Hakan Koyuncu ${ }^{4}$, Bilal Eryildirim ${ }^{5}$, \\ Bengi Gurses $^{2}$, Yavuz Bastug ${ }^{6}$, Bilger Erihan ${ }^{4}$
}

${ }^{1}$ Department of Radiology, Haydarpasa Numune Education and Research Hospital, ${ }^{2}$ Department of Radiology, Yeditepe University Medical Faculty, ${ }^{3}$ Department of Radiology Istanbul Education Hospital, ${ }^{4}$ Department of Urology, Yeditepe University Medical Faculty, ${ }^{5}$ Department of Urology Kartal Lutfi Kırdar Education and Research Hospital, ${ }^{6}$ Department of Urology Beykoz State Hospital, Istanbul, Turkey

\begin{abstract}
Objective: To monitor the impact of Shock Wave Lithotripsy (SWL) on the renal resisive index (RI) and to investigate the potential of the RI measurement for the estimation of the optimal duration between 2 SWL sessions. Material and methods: Thirty patients with single pelvis renalis stone were included. Participitants were grouped according to their age as group1 ( $<40$ years, mean age $36.2 \pm 3.9$ years) and group 2 ( $\geq 40$ years, mean age $55.4 \pm 6.5$ years). RI measurement was performed in of all patients prior to SWL. After SWL, RI was monitored daily until RI returned to their pre-SWL values. Results: The mean stone size was $28.97 \pm 3.62$ in group 1 and $10.08 \pm 4.67 \mathrm{~mm}$ in group $2(\mathrm{p}=0.077)$. Following SWL RI value of both goups increased and higher RI value was measured at $24^{\text {th }}$ hour as compared with their pre-SWL values $(p<0.001)$. In day 2 RI of the groups declined, but the differences were still statistically different from their pre-SWL RI values $(\mathrm{p}<0.001)$. However, on the third day, RI of group 1 was close to their pre-SWL level $(p=0.143)$. But, in group 2, RI value returned to their pre-SWL limits on day 4 ( $p=0.229)$. Conclusions: RI measurement gives important data regarding SWL related acute renal trauma and should be used as an US marker for recovery after SWL.
\end{abstract}

Keywords: color Doppler ultrasound, kidney, resistive index, Extracorporeal Shockwave Lithotripsy.

\section{Introduction}

Urolithiasis is one of the most common urological diseases, and extracorporeal shock wave lithotripsy $(\mathrm{SWL})$ is the method of choice in majority of cases [1,2]. Although it rarely leads to serious complications, SWL treatment is generally accepted as safe and has negligible side effects on long-term follow-up.

Received 02.12.2014 Accepted 10.01.2015

Med Ultrason

2015, Vol. 17, No 2, 175-179

Corresponding author: Esin Yencilek

Haydarpasa Numune Education and Research Hospital, Department of Radiology

Tibbiye Cad. No: 40 Uskudar, Istanbul, Turkey

Phone: +902165423232, Fax: +902163360565

E-mail: e.yenclek@yahoo.com
In urological practice, multiple SWL treatment sessions may be required depending on stone composition and patient-related factors. However, an "off period" (duration of stand-by time) between two SWL sessions is mandatory to protect the kidneys against repetitive shock wave-related trauma because acute adverse effects of SWL on renal morphology and function may occur despite its proven safety and efficacy during long-term followup. Therefore, urologists accept that there should be some duration between SWL sessions for kidney recovery. On the other hand, the ideal time interval between two SWL applications is not well known and remains under debate in the field of urology. It is not known exactly when the acute effect of shock waves on renal parenchyma returns to its normal limits. This question should be answered to estimate the ideal "off period" duration between sessions. 
Although some clinical experience with limited evidence indicates the feasibility of repeated SWL sessions within 1 day for ureteral stone, the interval required between repeated SWL sessions for renal stone treatment remains unknown [3]. Therefore, there is no consensus about the duration between SWL sessions. The "off period" varies between 5 days and 2 weeks in daily urological practice depending on urologist's or SWL technician's preference.

There are numerous radiologic reports concerning the impact of SWL on renal morphology and function [2,4-7]. Some of the studies focusing on the immediate vascular supply and total effective renal plasma flow of the kidneys indicated a transient decrease in these functions after SWL [4-7]. However, none of the radiological tools have been until now used to investigate the interval required between two SWL sessions. Measurement of the renal arterial resistive index (RI) of the kidney is a recently applied successful radiological tool that can reveal the resistance of intrarenal arteries, indirectly demonstrating effective renal blood flow [8-10]. Recently, it was shown that shock waves may decrease renal blood flow, which can lead to transient impairments in renal function after SWL [11].

In the present study, we aimed to evaluate and monitor renal RI changes after high-energy shock wave application in patients with pelvis renalis stones by using color Doppler ultrasonography (CDU), to show the ability of daily RI measurement during post-SWL follow-up, and to find the safe time interval between the first and second SWL sessions.

\section{Material and methods}

Adult patients with normal laboratory results (normal liver and kidney function tests, prothrombin time, activated partial thromboplastin time), no urinary tract infection, and a radiologically proven normal urinary system (no congenital anomaly, no obstruction) were enrolled in the study after approvel by the local ethics committee and providing written informed consent. Patients older than 18 years of age and having unilateral single pelvis renalis stone smaller than $20 \mathrm{~mm}$ were included for the study. The diagnosis of calculi was confirmed by noncontrast computed tomography (CT) and contrast enhanced images were taken to evaluate the urinary system anatomy in same CT session. Multiple stones, any kidney lesion other than stone, history of previous stone treatment (SWL or surgery), metabolic disease threatening kidney function such as hypertension or diabetes, obstructive stone, and body mass index $>30$ were the exclusion criterias of the study. All participants underwent a physical exam, urine culture to exclude urinary tract infection, and routine biochemical tests. Then, patients were grouped according to age into group 1 ( $<40$ years) and group 2 ( $\geq 40$ years).

Shock wave monotherapy was performed by using an electrohydraulic lithotriptor under analgesia that was achieved by intramuscular diclofenac sodium prior to SWL therapy. Upper limit for SW recieved by patients was 3000 at a frequency of $1-1.5 \mathrm{~Hz}$ and $18 \mathrm{kV}$ for the first SWL therapy session. The energy was increased in a stepwise manner with a pulse rate of $80 /$ minute to the maximum intensity after $500 \mathrm{SW}$.

Before the SWL session, the patients were evaluated using renal CDU (General Electric, Lociq 9, Milwaukee, USA) to measure their interlobar or arcuate RI. RI values of the upper, middle, and lower parts of the kidney were measured, and the arithmetic means of the three values were defined as the RI of the kidney. The RI of the contralateral kidney was also assessed using the same protocol. After the SWL therapy, all patients were followed for a daily RI measurement until their RI values returned to their pre-SWL limits. The daily RI values of the groups were compared with each other and their pre-SWL RI values.

Statistical Package for the Social Sciences (SPSS) version 17 was used for the data analysis. Repeated measures analysis of variance, paired $t$ tests, chi square test, and Mann Whitney-U tests were used for the statistical analysis. Values of $p<0.05$ were considered statistically significant at the $95 \%$ confidence level.

\section{Results}

Thirty patients were included in the study. Most of the stones in our study were on the left side (12 in the right and 18 in the left kidney). Table I represents the patients' demographic characteristics according to groups.

Before SWL monotherapy, RI values of the treated and nontreated kidneys were close to each other within the groups ( $\mathrm{p}=0.192$ group $1, \mathrm{p}=0.347$ group 2 ). In the treated kidneys, the RI value increased significantly after SWL session in both groups compared to their pre-SWL values $(p<0.001)$. Although a slight decrease was seen on day 2 the mean RI values of both groups remained high comparing with their corresponding pre-SWL RI values $(p<0.001)$. However, on day 3 , the mean RI value of group 1 was not significantly different from their pre-SWL value $(\mathrm{p}=0.143)$, and the difference in $3^{\text {rd }}$ day RI values between the two groups (group 1 and 2) was statistically significant $(p<0.001)$. The mean RI value of group 2 returned to the normal limit (pre-SWL RI) on day $4(\mathrm{p}=0.229)$, and the difference between group 1 and 
Table 1. Demographic charecteristics of the Younger (Group1) and Older (Group 2) gorups.

\begin{tabular}{llll}
\hline & Group 1 & Group 2 & p value \\
\hline Age (years) & $36.2 \pm 3.9$ & $55.4 \pm 6.5$ & $<0.001$ \\
Female & $3(27.3 \%)$ & $6(31.6 \%)$ & 0.064 \\
Male & $8(72.7 \%)$ & $13(68.4 \%)$ & 0.071 \\
BMI & $26.2 \pm 4.2$ & $28.3 \pm 3.0$ & 0.085 \\
Kreatinin (mg/dl) & $0.97 \pm 0.3$ & $1.08 \pm 0.7$ & 0.261 \\
BUN & $13.2 \pm 3.8$ & $15.7 \pm 4.7$ & 0.063 \\
Stone size (mm) & $8.97 \pm 3.62$ & $10.08 \pm 4.67$ & 0.077 \\
SWL duration (min) & $45.1 \pm 5.8$ & $46.9 \pm 8.8$ & 0.125 \\
SBP (mmHg) & $110 \pm 10.5$ & $128 \pm 15.2$ & 0.074 \\
DBP (mmHg) & $66 \pm 4.8$ & $72 \pm 9.9$ & 0.088 \\
Number of Shock Waves & $2859 \pm 100$ & $2921 \pm 57$ & 0.095 \\
Total Energy, kW & $2.98 \pm 0.49$ & $3.09 \pm 0.45$ & 0.182 \\
\hline
\end{tabular}

SBP: Systolic Blood Pressure; DBP: Diastolic Blood Pressure; min: Minute; mm: milimeters

Table II. The values of RI in treated and non-treated kidneys before and after SWL session

\begin{tabular}{llllllll}
\hline Groups & & pre-SWL & 24th hrs & 2ndday & 3rd day & 4th day & p \\
\hline Treated kidney & Group 1 & $0.588 \pm 0.11$ & $0.679 \pm 0.07$ & $0.643 \pm 0.11$ & $0.597 \pm 0.14$ & $0.592 \pm 0.08$ & $<0.001$ \\
& Group 2 & $0.596 \pm 0.04$ & $0.683 \pm 0.13$ & $0.661 \pm 0.11$ & $0.642 \pm 0.22$ & $0.601 \pm 0.09$ & $<0.001$ \\
\multirow{2}{*}{ Non-treated kidney } & Group 1 & $0.581 \pm 0.09$ & $0.583 \pm 0.41$ & $0.579 \pm 0.22$ & $0.592 \pm 0.14$ & $0.575 \pm 0.32$ & 0.09 \\
& Group 2 & $0.585 \pm 0.05$ & $0.593 \pm 0.13$ & $0.591 \pm 0.08$ & $0.586 \pm 0.16$ & $0.590 \pm 0.11$ & 0.12 \\
\hline
\end{tabular}

2 was not significant by day $4(\mathrm{p}=0.154)$. Evaluation of $\mathrm{RI}$ in the contralateral nontreated kidneys revealed no significant change in RI values of pre-SWL versus earlyand late-term post-SWL values in both groups (table II).

\section{Discussions}

Renal stones, one of the most common urological diseases, should be treated by SWL according to urological guidelines. SWL treatment success depends on technical, stone, and patient-related factors, and repeated sessions are frequently needed for stone disintegration. However, an "off period" is suggested between SWL sessions to protect the renal parenchyma against repetitive trauma caused by the shock waves [3]. The underlying reason for this suggestion depends on the fact that SWL causes acute trauma to the treated kidney parenchyma and its vasculature. On the other hand, the optimal duration of an "off period" between SWL sessions is debatable in urology. In present study, we aimed to show the capability of RI slope after SWL therapy to monitor shock wave-related trauma to kidneys with pelvis renalis stones in order to estimate the optimal "off period" time interval by age.

It is well known that shock wave-related high energy can potentially impair renal function. In the literature, the effects of shock waves on the morphology and function of treated kidneys have been subjected to several clinical and experimental studies [2,4-6]. Many were concen- trated to clarify the short- or long-term effect of the shock waves on the renal parenchyme and the vast majority of the studies showed that the acute effect of SWL on the renal tissues was temporary due to its compensatory mechanism [12]. Today it is accepted that SWL treatment for renal stones is safe and reliable on long-term follow-up.

Although many studies have shown the efficacy and reliability of SWL, the optimal duration of the "off period" between SWL sessions has yet to be determined. This is an unanswered traditional question in urology because estimating the optimal safe time is important to protecting the renal parenchyma against repetitive shock wave-related trauma. Although some clinical experience with low degree of evidence indicates the possibility of repeating SWL session within 1 day for ureteral stones, we found no clinical study or experience concerning this aspect [2]. The degree of impact of shock waves on ureteral and renal tissue has not been largely studied. However, it is expected that renal tissue is more prone to SWL-related damage owing to its highly vascular thick parenchyma compared to that of ureteral tissue. Therefore, it may be logical to think that the "off period" for renal tissues in renal stone lithotripsy should be longer than that of ureteral tissue. The present study was the first to measure daily RI to demonstrate its decreases. Here we tried to monitor the slope of the RI decrease after SWL therapy and to develop an understanding of the time required for RI values of treated kidneys to return to normal limits. 
Some radiological tools that were used to measure total effective renal plasma flow of the kidneys indicated a transient decrease in renal function after SWL $[4,6,7]$. Also, numerous radiological studies have shown the potential usefulness of imaging techniques in the assessment of early renal damage $[7,13,14]$. Almost all radiological studies in the literature have focused on the limitations of radiologic tools to understand SWL-related changes. Among these tools, CDU is easy to apply, cost effective, and has the potential to determine renal function and kidney microcirculation [8,9,15-17]. CDU was recently used as a noninvasive method to estimate renal function and has been used to obtain functional information after SWL sessions.

Although some authors showed no change in kidney RI, most studies reported increased RI values after SWL sessions [18-20]. The discrepancies between studies might be due to study group differences and study population heterogeneity. In some series, RI values were studied in stone-related obstructed cases but others were measured RI in patients without obstruction. Although there is some contraversies about the impact of obstruction on RI value, obstruction may have potential to affect renal vascular resistance and causes renal perfusion changes [21-24]. Also, patients in the different studies were subjected to different numbers of shock waves. This may also help explain differences among studies showing the relationship between RI and SWL in different series. Therefore, here we studied non-obstructed cases to exclude the possible impact of obstruction on renal tissues. Additionally, the number of shock waves used in our series was similar between groups.

Currently, an increase in RI values is expected after SWL therapy, which has been confirmed in many studies, though there are a few exceptions $[11,18]$. In the present study, we found higher RI values after SWL sessions in patients in both groups, comparable to that reported previously in literature. Increases in RI values were similar between groups as well. In the literature, increases in RI are time-dependent and the acute effect of the shock waves disappears after some time, most likely due to compensatory mechanisms of the kidney [22]. In agreement with the literature, the RI values in our series returned to almost pre-SWL values several days after SWL treatment. High RI values of the treated kidney lasted from a few days up to day 4 after SWL depending on age group.

The relationship between age and increase in RI values is also well studied. Almost all of the results showed that the increase in RI after SWL sessions is not related to age [25]. We also found high RI values immediately after SWL treatment in both groups. However, until now, no clinical study has examined the RI decrease on a day-to-day basis; in fact, almost all previous studies measured RI over a predetermined time interval, which cannot determine the exact day of RI recovery to normal values (pre-SWL values). Therefore, we monitored the daily RI of each treated kidney and found that the mean RI values returned to their pre-SWL levels on day 3 in the younger group ( $<40$ years) and on day 4 in the older group ( $>40$ years). We do not have data to explain the underlying reason for the differences in RI recovery between age groups. However, in the literature, higher RI values in older versus younger patients were explained by decreased elasticity in the renal tissues of elderly patients [8]. This conclusion seems logical but should be confirmed by preclinical studies.

Limited studies have evaluated the untreated contralateral kidney in response to $\mathrm{SW}$ and data shows a debate in this issue. Some authors found no significant changes in RI values of untreated kidneys versus treated kidneys [26]. In contrast, Mitterberger et al found higher RI measurements after SWL in the contralateral kidney [8]. We found no significant increase in RI values of untreated kidneys after SWL. The difference in the literature can be explained by group heterogeneity, different numbers of shock waves, or the use of medication before SWL in the different study groups.

Although our data confirm the ability of RI to monitor renal blood flow and renal parenchymal RI and assess the optimal "off period" between the first and second SWL sessions, this study has some limitations. First, its main limitation is the few subjects included. Second, the quantitative data obtained from the CDU could not be compared with the histological findings; therefore, the exact relationship between RI value and renal tissue resistance or transient functional alteration after SWL is obscure. Lastly, two experienced radiologists measured the RI of the patients due to multicentricity of the study. However, despite the absence of such data, our results demonstrate the ability of RI to estimate the optimal "off period" in patients with renal stones, and that RI decreases with age among patients receiving SWL.

\section{Conclusions}

RI gives an idea regarding SWL related acute renal trauma and should be used as an US marker for recovery after SWL and for prediction in estimating the optimal period between two SWL procedures. However, further studies including more patients are required.

Conflict of interest: none 


\section{References}

1. Bozgeyik Z, Kocakoc E, Sonmezgoz F. Diffusion-weighted MR imaging findings of kidneys in patients with early phase of obstruction. Eur J Radiol 2009; 70: 138-141.

2. Muller MF, Prasad PV, Bimmler D, Kaiser A, Edelman RR. Functional imaging of the kidney by means of measurement of the apparent diffusion coefficient. Radiology 1994; 193: 711-715.

3. Türk C, Knoll T, Petrik A, Sarica K, Skolarikos A, Straub M, Seitz C. EAU Guidelines on Urolithiasis 2014. Available at http://uroweb.org/wp-content/uploads/22-Urolithiasis_LR.pdf.

4. Laissy JP, Menegazzo D, Dumont E, et al. Hemodynamic effect of iodinated high-viscosity contrast medium in the rat kidney. Invest Radiol 2000; 35: 647-652.

5. Chan JH, TsuiEY, Luk SH, et al. MR diffusion-weighted imaging of kidney: differentiation between hydronephrosis and pyonephrosis. Clin Imaging 2001; 25: 110-113.

6. Le Bihan D, Delannoy J, Levin RL. Temperature mapping with MR imaging of molecular diffusion: application to hyperthermia. Radiology 1989; 171: 853-857.

7. Zhang H, Prince MR. Renal MR angiography. Magn Reson Imaging Clin N Am 2004; 12: 487-503.

8. Aoki Y, Ishitoya S, Okubo K, et al. Changes in resistive index following extracorporeal shock wave lithotripsy. Int J Urol 1999; 6: 483-492.

9. Nazaroglu H, Akay AF, Bükte Y, Sahin H, Akkus Z, Bilici A. Effects of extracorporeal shock-wave lithotripsy on intrarenal resistive index. Scand J Urol Nephrol 2003; 37: 408-412.

10. Tatar IG, Teber MA, Ogur T, Kurt A, Hekimoglu B. Real time sonoelastographic evaluation of renal allografts in correlation with clinical prognostic parameters: comparison of linear and convex transducers according to segmental anatomy. Med Ultrason 2014; 16: 229-235.

11. Knapp R, Frauscher F, Helweg G, et al. Age-related changes in resistive index following extracorporeal shock wave lithotripsy. J Urol 1995; 154: 955-958.

12. Strohmaier WL, Carl AM, Wilbert DM, Bichler KH. Effects of extracorporeal shock wave lithotripsy on plasma concentrations of endothelin and renin in humans. J Urol 1996; 155: 48-51.

13. Riccabona M, Ruppert-Kohlmayr A, Ring E, Maier C, Lusuardi L, Riccabona M. Potential impact of pediatric MR urography on the imaging algorithm in patients with a functional single kidney. AJR Am J Roentgenol 2004; 183: 795-800.

14. Grenier N, Basseau F, Ries M, Tyndal B, Jones R, Moonen C. Functional MRI of the kidney. Abdom Imaging 2003; 28: $164-175$.
15. Kurt S, Tokgöz Ö, Tokgöz H, Voyvoda N. Evaluation of effects of Extracorporeal Shock Wave Lithotripsy on renal vasculature with Doppler ultrasonography. Med Ultrason 2013; 15: 273-277.

16. Abd Ellah M, Kremser C, Pallwein L, et al. Changes of renal blood flow after ESWL: assessment by ASL MR imaging, contrast enhanced MR imaging, and renal resistive index. Eur J Radiol 2010; 76: 124-128.

17. Ghadirpour A, Tarzamni MK, Naghavi-Behzad M, AbediAzar S, Koushavar H, Nezami N. Renal vascular Doppler ultrasonographic indices and carotid artery intima-media thickness in diabetic nephropathy. Med Ultrason 2014; 16 : 95-99.

18. Beduk Y, Erden I, Gogus O, Sarica K, Aytac S, Karalezli G. Evaluation of renal morphology an vascular function by color flow Doppler sonography immediately after extracorporeal shock wave lithotripsy. J Endourol 1993; 7: 457-460.

19. Zolfaghari A, Ghadirpour A, Tarzamni MK, Goldust M, Mirabad MR, Nezami N. Renal vascular Doppler resistance after extracorporeal shock wave lithotripsy. Ren Fail 2013; 35: 686-690.

20. Mitterberger M, Pinggera GM, Neururer R, et al. Multimodal evaluation of renal perfusional changes due to extracorporeal shock wave lithotripsy. BJU Int 2008; 101: 731-735.

21. Gurel S, Akata D, Gurel K, Ozmen MN, Akhan O. Correlation between the renal resistive index (RI) and nonenhanced computed tomography in acute renal colic: how reliable is the RI in distinguishing obstruction? J Ultrasound Med 2006; 25: 1113-1120.

22. Opdenakker L, Oyen R, Vervloessem I, et al. Acute obstruction of the renal collecting system: the intrarenal resistive index is a useful yet time dependent parameter for diagnosis. Eur Radiol 1998; 8: 1429-1432.

23. Bertolotto M, Moro U, Gioulis E, Lodolo C, Lissiani A. Changes of renal resistive index in response to hydration and diuretic administration in normal subjects and in patients with small ureteral stone. J Ultrasound Med 1999; 18: 819-825.

24. Lee HJ, Kim SH, Jeong YK, Yeun KM. Doppler sonographic resistive index in obstructed kidneys. J Ultrasound Med 1996; 15: 613-618.

25. Mohseni MG, H Khazaeli M, Aghamir SM, Biniaz F. Changes in intrarenal resistive index following electromagnetic extracorporeal shock wave lithotripsy. Urol J 2007; 4: 217-220.

26. Juan YS, Huang CH, Wang CJ, et al. Predictive role of renal resistance indices in the extracorporeal shock-wave lithotripsy outcome of ureteral stones. Scand J Urol Nephrol 2008; 42: 364-368. 